\title{
Message from the Editor-in-Chief
}

\author{
YOICHI TAKENAKA ${ }^{1, a)}$
}

This is the tenth volume of IPSJ Transactions on Bioinformatics (TBIO) after its renewal in 2008. The first issue of TBIO was published in November 2006 as the first English Transactions from Information Processing Society of Japan (IPSJ). After publishing four issues, TBIO restarted with Volume 1 from November 2008, according to the renewal of all the IPSJ journals and transactions.

TBIO is an open access journal publishing research articles in bioinformatics, computational biology, and any related topics in various fields, including information science, statistics, mathematics and bioscience. Our common goal is understanding of the principles of life. We publish both articles the aim directly at the goal and those that aim to provide methods for achieving the goal. To reach wider audience in various fields, this journal was started as English journal. Moreover, it is an open access journal via the J-Stage system (http://www.jstage.jst.go.jp/browse), and the authors can circulate their results widely. This publication is edited and financed by the Special Interest Group of Bioinformatics (SIGBIO), IPSJ.

The most distinctive characteristic of this publication is its evaluation criteria for reviews. We evaluate papers based on the merits of them, unlike the conventional review systems based on points on various aspects of papers. Papers which present either enough novelty or enough usefulness with a competent level of English writing will be considered for publication. Papers may also be evaluated on their future potential. Another important characteristic of this publication is its short review period, most articles being reviewed within a month. The submission guidelines of this journal are at http://www.ipsj.or.jp/katsudou/sig/sighp/bio/IPSJTBIO_e.html (Engish) and http://www.ipsj.or.jp/katsudou/sig/sighp/bio/tbio. html (Japanese). Each manuscript submitted to TBIO is reviews by at least two referees.

TBIO accepts three kinds of papers: original papers, survey papers and database/software papers. The topics covered by TBIO includes, but not limited to: sequence analysis, evolutionary tree analysis, prediction and analysis of protein/RNA structures, molecular dynamics, analysis of various kinds of biological networks, gene polymorphism data analysis, cell simulation, neuro-informatics, methylation analysis and systems biology. The results of these papers have been or will be presented at IPSJ-SIGBIO meetings, whose information is available

\footnotetext{
Editor-in-Chief

Department of Bioinformatic Engineering, Graduate School of Information Science and Technology, Osaka University, Suita, Osaka 565-0871, Japan

a) takenaka@ist.osaka-u.ac.jp
}

at http://www.ipsj.or.jp/katsudou/sig/sighp/bio/.

I thank all the authors of this volume and hope that readers enjoy reading these papers. I would like to invite potential authors to submit any types of paper in bioinformatics and/or computational biology. I look forward to receiving more and more submissions as well as seeing many presentations at IPSJ-SIGBIO meetings. 